\title{
Hematopoietic Stem Cell Transplantation: The Quality Matters
}

\section{Khawaja Husnain Haider*}

Department of Basic Sciences, Sulaiman AIRajhi Colleges, Saudi Arabia

\section{Keywords: Hematopoietic; Platelets; Stem cells; Transplantation}

List of Abbreviations: EGP: Epidermal Growth Factor; FBS: Fetal Bovine Serum; FGF-2: Fibroblast Growth Factor-2; HGF-1: Hepatocyte Growth Factor-1; HSCs: Hematopoietic Stem Cells; IGF-1: Insulin-like Growth Factor; IL: Interleukin; MSCs: Mesenchymal Stem Cells; PBSCs: Peripheral Blood Stem Cells; PDGF: Platelet Derived Growth Factor; PRP: Platelet Rich Plasma; TGF- $\beta$ : Transforming Growth Factor- $\beta$; VEGF: Vascular Endothelial Growth Factor

\section{Letter to Editor}

Transplantation of Hematopoietic Stem Cells (HSCs) derived from the bone marrow, umbilical cord blood, and peripheral blood has been in the clinical practice since decades as an effective therapeutic modality for both benign as well as malignant hematological and non-hematological disorders. Nevertheless, prognosis after HSC transplantation is influenced by multitude of factors that are determinant of the quality of the cell graft, encompassing from HSC source and composition to the post-thawing recovery rate and viability of the cryopreserved cells. Cryopreservation is integral to transplantation protocols when cord blood derived HSCs or autologous HSCs are sourced during cell transplantation procedure. Hence, optimization of the cryopreservation methods has remained an area of intense research until the recent past. Accessibility to the optimized cryopreservation protocols currently warrants long-term high level viable recovery of HSCs with little alteration in engraftment rate, stemness and functionality [1-3]. Nevertheless, there is an obvious dearth of literature regarding the composition of the cell graft especially with respect to the presence of platelets that possibly influence the recovery and viability of the cryopreserved HSCs and, their engraftment and function posttransplantation. Valentini et al. have addressed this interesting aspect of HSCs research in their recently published study to demonstrate that the higher concentration of platelets in the Peripheral Blood Stem Cells (PBSCs) negatively impacts the survival and recovery rates of the peripheral blood derived autologous HSCs after cryopreservation [4]. Prior studies have also reported that CD34+ cell survival in the cryopreserved cell preparations was inversely related with the ratio between CD34+ cells and the total nucleated cells including white blood cells and neutrophils; however, the effect of platelets concentration was ignored in the study [5]. Supported by the remarkable experimental data in which increasing concentrations of platelets were cryopreserved with HSCs, Valentini et al. have shown adverse effects of higher platelet number on the viable HSC recovery [4]. Therefore, the authors have recommended that platelets should be removed before cryopreservation of HSCs or alternatively the cells ought to be immediately washed after thawing to minimize the unwarranted influence of the platelet-derived factors in terms of their viability and engraftment potential. These are interesting observations that need to be substantiated further to establish the mechanism of the harmful influence of the platelets on HSCs during cryopreservation.

Proteomic analysis of platelets shows their richness in bioactive molecules including the clotting factors, growth factors, i.e., PDGF, TGF-1 $\beta$, IGF-1, HGF-1, VEGF, FGF2, and various interleukins, i.e., IL1, Il4, IL6, IL10, IL13. Hence, the platelet derived preparations including the platelet lysate and the releasate from activated platelets are currently being explored as a substitute to the Fetal Bovine Serum (FBS) to support in vitro culture and expansion of stem/ progenitor cells, overcome their senescence and promote their rejuvenation [6-7]. On the same note, Platelet Rich Plasma (PRP) which is affluent in platelet derived growth factors is being used to support stem/progenitor cell proliferation and differentiation in vitro as well as post-transplantation [8]. However, the composition of PRP derived lysate and releasate is determined by its cellular components and may differ due to contaminant leukocyte population [9]. Repeated freeze-thaw cycles (3-5 cycles) optimally achieve growth factor-rich lysate preparation with clinical relevance [10]. Similarly, platelet gel preparations are equally affluent in growth factors and cytokines. It is pertinent to mention that the platelets obtained from different tissue sources are diverse in their growth factor proteome [11]. A direct proteomic comparison of the cord blood and peripheral blood derived platelet releasate, lysate and platelet gel preparations showed that the two platelet sources diverged in the levels of inflammatory and angiogenic growth factors [11]. In vitro treatment of stem/ progenitor cells, i.e., mesenchymal stem cells (MSCs) from the bone marrow and the adipose tissue, with platelet derived preparations significantly support their culture characteristics [12]. However, the molecular mechanism underlying the salutary effects of platelet-derived preparations on stem/progenitor cells remains less well-studied and warrants in-depth investigation. It is generally believed that the bioactive molecules from the platelets act on their respective membrane receptors on the HSCs to initiate signaling pathways that regulate various cell functions. Contrarily, the observed detrimental effect of the platelets on the cryopreserved HSCs is intriguing and may be attributed to the release of pro-inflammatory cytokines by the peripheral blood platelets [4]. These data may have widespread implications in the regenerative medicine where both bone marrow as well as peripheral blood derived HSCs have been extensively studied for their reparability [13]. HSCs are multipotent and have the ability to differentiate into hematopoietic as well as non-hematopoietic lineages including the cardiomyogenic and vasculogenic lineages [14]. HSCs also secrete copious amounts of growth factors and cytokines as a part of their paracrine activity to impart cytoprotection besides providing chemical cues for the intrinsic pool of resident stem cells to home-in to the injured site and participate in the repair process [15]. Alternatively, treatment with paracrine factors suppresses the levels of pro-inflammatory cytokines [16]. Therefore, an important application of HSCs has been for the repair of the infarcted heart during the clinical studies in the human patients [17]. The limited

*Corresponding authors: Khawaja Husnain Haider, Professor, Molecular and Cellular Pharmacology (Stem cells and Gene Therapy), Department of Basic Sciences, Sulaiman AIRajhi Colleges P.O. Box 777, Al Bukairiyah-51941, Saudi Arabia, Tel: 06-3355555 Ext. -7704; E-mail: kh.haider@sr.edu.sa

Received May 22, 2017; Accepted June 01, 2017; Published June 09, 2017

Citation: Haider KH (2017) Hematopoietic Stem Cell Transplantation: The Quality Matters. J Stem Cell Res Ther 7: 390. doi: 10.4172/2157-7633.1000390

Copyright: (c) 2017 Haider $\mathrm{KH}$. This is an open-access article distributed under the terms of the Creative Commons Attribution License, which permits unrestricted use, distribution, and reproduction in any medium, provided the original author and source are credited. 
Citation: Haider KH (2017) Hematopoietic Stem Cell Transplantation: The Quality Matters. J Stem Cell Res Ther 7: 390. doi: 10.4172/21577633.1000390

success achieved during these clinical studies has been generally ascribed to the inability of HSCs to cross the lineage barrier and adopt cardiac phenotype. However, most of the studies published in this regard actually ignored the quality of the cell preparation [18]. Given that the presence of the contaminated red blood cells, the total nucleated cell population including the white blood cells, and the platelets significantly interfere with survival of the cryopreserved HSCs as well as their engraftment potential after transplantation, it is imperative that the HSCs preparations used in the clinical trials for cell-based therapy should be assessed for quality in terms of the presence of contaminating cell populations. This is even more important when the un-expanded HSCs are used for transplantation until protocols for in vitro expansion of HSCs become optimized [19].

\section{References}

1. Broxmeyer HE, Srour EF, Hangoc G, Cooper S, Anderson SA et al. (2003) High-efficiency recovery of functional hematopoietic progenitor and stem cells from human cord blood cryopreserved for 15 years. Proc Natl Acad Sci USA 100: 645-650. [PubMed]

2. Berz D, McCormack EM, Winer ES, Colvin GA, Quesenberry PJ (2007) Cryopreservation of hematopoietic stem cells. Am J Hematol 82: 463- 472. [PubMed]

3. Lee HR, Song EY, Shin S, Roh EY, Yoon JH, et al. (2014) Quality of cord blood cryopreserved for up to 5 years. Blood Res 49: 54-60. [PubMed]

4. Valentini CG, Bianchi M, Orlando N, Autore F, lachininoto MG, et al. (2017) Graft composition and post-thawing cell viability influence the hematopoietic recovery in autologous hematopoietic stem cell transplantation. J Stem Cell Res Ther 7: 379.

5. Castelhano MV, Reis-Alves SC, Vigorito AC, Rocha FF, Pereira-Cunha FG, et al. (2013) Quantifying loss of CD34+ cells collected by apheresis after processing for freezing and post-thaw. Transfus Apher Sci 48: 241-246. [PubMed]

6. Griffiths S, Baraniak PR, Copland IB, Nerem RM, McDevitt TC (2013) Human platelet lysate stimulates high-passage and senescent human multipotent mesenchymal stromal cell growth and rejuvenation in vitro. Cytotherapy 15 1469-1483. [PubMed]
7. Kinzebach S, Dietz L, Klüter H, Thierse H-J, Bieback K (2013) Functional and differential proteomic analyses to identify platelet derived factors affecting ex vivo expansion of mesenchymal stromal cells. BMC Cell Biol 14: 48. [PubMed]

8. Tobita M, Tajima S, Mizuno H (2015) Adipose tissue-derived mesenchymal stem cells and platelet-rich plasma: stem cell transplantation methods that enhance stemness. Stem Cell Res Ther 6: 215. [PubMed]

9. Sundman EA, Coley BJ, Fortier LA (2011) Growth factor and catabolic cytokine concentrations are influenced by the cellular composition of platelet-rich plasma. Am J Sports Med 39: 2135-2140. [PubMed]

10. Strandberg G, Sellberg F, Sommar P, Ronaghi M, Lubenow N (2017) Standardizing the freeze-thaw preparation of growth factors from platelet lysate. Transfusion 57: 1058-1065. [PubMed]

11. Parazzi V, Lavazza C, Boldrin V, Montelatici E, Pallotti F, et al. (2015) Extensive characterization of platelet gel releasate from cord blood in regenerative medicine. Cell Transplant 24: 2573-2584. [PubMed]

12. Astori G, Amati E, Bambi F, Bernardi M, Chieregato K, et al. (2016) Platelet lysate as a substitute for animal serum for the ex-vivo expansion of mesenchymal stem/stromal cells: present and future. Stem Cell Res Ther 7: 93. [PubMed]

13. Li MD, Atkins H, Bubela T (2014) The global landscape of stem cell clinical trials. Regen Med 9: 27-39. [PubMed]

14. Haider HKh, Ashraf M (2005) Bone marrow stem cell transplantation for cardiac repair. Am J Physiol Heart Circ Physiol 288: H2557-H2567. [PubMed]

15. Lei Y, Haider HKH (2010) Pararining the heart with stem cells. In: Stem cells- from drug to drug discovery. Haider HKh (ed) Walter de Gruyter $\mathrm{GmbH}$, Germany, pp: 176-203.

16. Haider KH, Aziz S (2017) Paracrine hypothesis and cardiac repair. Int J Stem Cell Res Transplant 5: 265-267.

17. Trounson A, Thakar RG, Lomax G, Gibbons D (2011) Clinical trials for stem cell therapies. BMC Med 9: 52. [PubMed]

18. Shahid MS, Lasheen W, Haider KH (2016) The modest outcome of clinical trials with bone marrow cells for myocardial repair: is the autologous source of cells the prime culprit? J Thorac Dis 8: E1371-E1374. [PubMed]

19. Park B, Yoo KH, Kim C (2015) Hematopoietic stem cell expansion and generation: the ways to make a breakthrough. Blood Res 50: 194-203. [PubMed] 